Case report

\title{
Dealing with the jugular foramen; A case of dumbbellschwannoma
}

BalasubramaniumA ${ }^{1}$ (D), Daminda DADG ${ }^{2}$, Rupasinghe $\mathrm{RT}^{3}$, Zakeer $\mathrm{R}^{4}$

${ }^{1}$ Registrar in ENT, Teaching Hospital Anuradhapura, Sri Lanka

${ }^{2}$ Consultant ENT surgeon, Teaching Hospital Anuradhapura, Sri Lanka

${ }^{3}$ Consultant ENT surgeon, Teaching Hospital Anuradhapura, Sri Lanka

${ }^{4}$ Consultant Radiologist, Teaching Hospital Anuradhapura, Sri Lanka

\begin{abstract}
A 57-year-oldpatient diagnosed witha right glomus jugularae was referred to the ENT unit TH/Anuradhapura for further management. Multi-disciplinary discussion and multi-modality imaging confirmed the diagnosis to be a jugular schwannoma. The Patient underwenttumour excision via a combined petro-occipital trans-sigmoidal approach withclose perioperative monitoring and post-operative rehabilitation. This article discusses the importance of a detailed history, examination and multimodality imaging for correct diagnosis of jugular foramen lesions which governs the selection of the surgical approach.
\end{abstract}

Key words: Interdisciplinary Health Team, Multimodal Imaging,Schwannoma

Copyright: () 2019 Balasubramanium A et al.

This is an open access article distributed under the Creative Commons Attribution License (CC-BY), This license lets others distribute, remix, tweak, and build upon the work, even commercially, as long as they credit the original author for the creation.

Funding: None

Competing interest: None

Correspondence: Dr. A. Balasubramanium (abarnnab@gmail.com )

(iD https://orchid.org/0000-0001-9244-1409 


\section{Case Report}

A 57-year-old patient with a diagnosis ofa right glomus jugularae tumour was referred to the ENT clinic at teaching hospital Anuradhapura for further management in February of 2017.

The Patient had noticed a painless lump on the right side of the neck eight years back. Hehad subsequently sought medical advice from different parts of the country and even from overseas which led to a MRI scan of the head and neckwhich reported tohave a glomus tumour. Hehad beenadvised regardingsurgical excisionbut he defaulted treatment after knowing the possible complications.The lump progressively increased in size causingsnoring. He also developed a voice change, right sided facial weakness and found difficulty in protruding the tongue. In 2016 December, he was admitted to the District General Hospital(DGH) Vavuniya with a history of aspiration pneumonia.During examination he had large right lateral pharyngeal mass with right vocal cord palsy. A CT of the head and neckdone in 2017 had revealed further enlargement and erosions of skull base tumour compared to the previous MRI done in 2011. On examination we found a large massextending down from rightside of the nasopharynx to hypopharynx. He also had right VI, VII, IX, X and XII cranial nerve palsies and 'mild' bilateral cerebellar signs.

A Multi-disciplinary team (MDT) comprising ENT, neurosurgery, vascular surgery,oncology and radiologyconcluded that this tumour has to be removed surgically.

MRI with MRA and MRV of the head and neck was requested to assess the vascularity of the tumour. The preliminary report of MRI revealed a tumourwith decreased vascularity, cystic changes and a fluid levelfavouring aSchwannomarather than a glomus jugularae (Images 1a). A CT angiogramof the head and neck was taken to further clarifythe diagnosis. Thisadditionally revealed that the schwannoma was likely arising from the $\mathrm{IX}^{\text {th }}$ and $\mathrm{X}^{\text {th }}$ cranial nerveswith both intracranial and extra cranial components. (Image 1b)

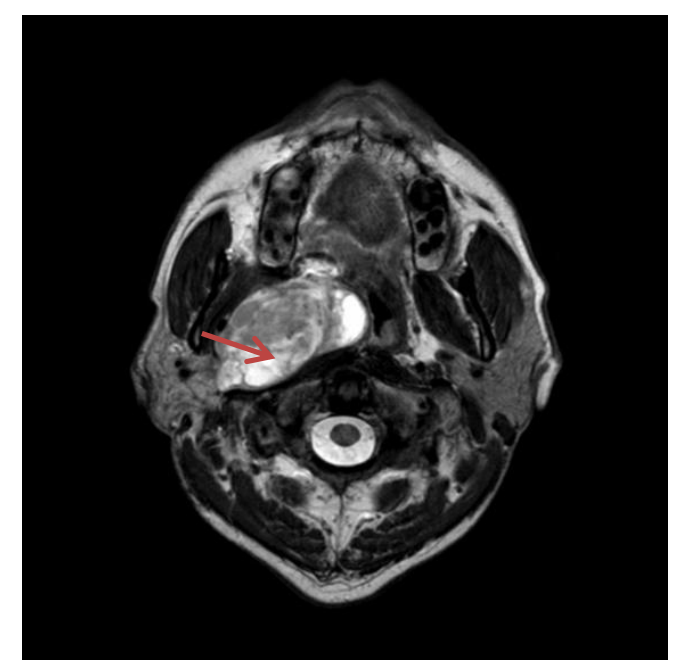

Image 1(a) - MRI, Axial View, Tumour Highlighted with a red pointer

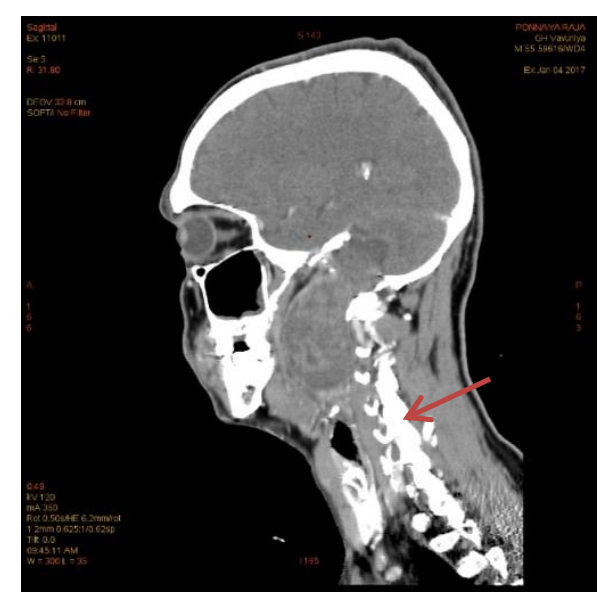

Image 1(b) - CT, Coronal view,Tumour Highlighted with a red pointer 
A decision was taken to go for a combined surgery (ENT and Neurosurgical teams). A Right petro-occipital trans-sigmoid(POTS) approach along with an elective tracheostomy was selected. The Tumour was removed completely.

Functional endoscopic evaluation of swallowing (FEES) carried out after one week showed aspiration. Swallowing therapy was started and it was planned to keep the nasogastric (NG) tube and cuffed tracheostomy tube until he adapted to swallowing.

On the eighth post-optative day he developed a cerebrospinal fluid (CSF) leak. Initially it was managed conservatively buta lumbar drain had to beplacedlater. Broad spectrum intravenous antibiotics were also started prophylactically. The NG tube and the tracheostomy tube were removed after surgery in 2 months and 5 months respectively.

Histology report revealed that the tumour was a Schwannoma.

\section{Discussion}

In clinical practice jugular foramen lesions are rare ${ }^{[1]}$. Dumbbell shape lesions are very rare presentations. The treatment options are surgical excision and/or radiation therapy or gamma knife. Surgery is challengingbecause of the regions complex anatomy and the potential for multiple complications.

Jugular foramen lesions are broadly classified into neoplastic and non-neoplastic lesions. In the neoplastic element paraganglioma is the most common. Schwannoma, meningioma and metastasis are lesscommon ${ }^{[2]}$.

This tumour canexpand either superiorly into the posterior cranial fossa or inferiorly into the parapharyngeal space ${ }^{[3]}$. Pellet et al modified the grading system of the Schwannoma which was originally developed by Kaye et al. Our patient had type D schwannoma.

As paraganglioma is the most common lesion in this regionand also one of the differential diagnoses for schwannoma, our patient was misdiagnosed initially as having glomus paraganglioma.

The clinical manifestations depend on the size and the anatomical location of the tumour.Our patient had a visible mass in right side of the oropharynx, which made him seek medical advice initially. He later developed progressive lower cranial nerve weakness on the right side.

Multimodality imaging is the most important investigation in the radiological differential diagnoses of jugular foramen lesions ${ }^{[2]}$. High resolution computer tomography (HRCT), MRI and angiography of brain will give maximum details of the lesion, which helps in the diagnosis and decision making on surgical treatment.

During the initial period of evaluation our patient underwent MRI and not CT. MRI images can be similar for both paraganglioma and schwannoma. In this regard, theCT scan is the investigation of choice to differentiate both lesions.(When we were initially investigating the patient, the radiologists queriedthe diagnosis and wanted to do CT head and neck with angiogram. Subsequently theyreported the lesion as Jugular foramen schwannoma.This diagnosis changed our management plan.)

Total tumour removal is the treatment of choice for jugular foramen tumours. The surgical approach for the lesion depends on the site of origin, size, extent and vascularity of the lesion ${ }^{[4]}$. Acombined approach retrosigmoidal craniotomy and a cortical mastoidectomy for intracranial portion and neck dissection for infratemporal portion were planned to remove the tumour completely with preservation of hearing.

Post-operative complications depend on the size, extent and vascularity of the tumour, pre-operative condition of the patient, choice of surgical approach and the skill of the surgeon. CSF lea and meningitis is a common problem after skull base surgery ${ }^{[3]}$. 
We expected an increased risk of aspiration for a transient period soon after the surgery as he had preexisting lower cranial nerve palsies, we explained and got pre-operative consent for a temporary tracheostomy and NG feeding. FEES after one week of surgery revealed aspiration andswallowing therapy was started. After 2 months he had some improvement in swallowing and the NG tube was removed with a dietary modification. Tracheostomydecanulation was done after 5 months of surgery.

\section{Conclusion}

A Detailed history, examination and multimodality imaging are important for the correct diagnosis of jugular foramen lesions. MDT discussion, combined surgery and close peri-operative monitoring will reduce morbidity.

\section{References:-}

1. Cebeci S. et.al, Jugular foramen schwannoma-Case report. International journal of Pediatric Otorhinolaryngology Extra.December 2011;6(4):322-324

2. www.thamburaj.com-neurosurgery on the web / jugular foramen lesions.htm (cited on 21.11.17)

3. Rayappa C., Jugular foramen tumours. Otolaryngology clinics: An International journal January-April 2011;3(1):15-23

4. Baker B., The jugular foramen Schwannomas: Review of the large surgical series. $J$ Korean NeurosurgSoc Nov 2008; 44(5): 285-294 